\title{
Utility of Intrathoracic Impedance Monitoring in Pediatric and Congenital Heart Disease
}

\author{
MARTIN J. LAPAGE, M.D., JOHANNES VON ALVENSLEBEN, M.D., \\ MACDONALD DICK, M.D., GERALD SERWER, M.D., and DAVID J. BRADLEY, M.D.
}

From the Department of Pediatrics and Communicable Diseases, Division of Pediatric Cardiology, University of Michigan, Ann Arbor, Michigan

Background: The utility of cardiac device-based intrathoracic monitoring (OptiVol, Medtronic Inc., Minneapolis, MN, USA) for congestive heart failure (CHF) exacerbation has not been evaluated in pediatric or congenital heart disease patients.

Methods: This was a retrospective study of all patients at a single center with an endocardial OptiVol capable device. OptiVol index values were collected in 2-week bins from January 2007 to December 2010. The clinical outcomes were CHF exacerbation defined as hospitalization or medication change for CHF and device-treated ventricular arrhythmia based on remote or in-office device interrogation. Clinical and OptiVol data were collected by separate investigators blinded to the other parameter. OptiVol data were correlated to the clinical outcomes to determine sensitivity and predictability for multiple threshold values in the entire cohort and pediatric and congenital subgroups.

Results: Forty-seven patients were included. A total of 1,106 months of OptiVol data were collected. Median age of the cohort was 18 years (range 6-58 years). There were 23 pediatric, median age 13 years (range 6-16), at device implant, and 18 patients, median age 31 years (range 13-58), considered at risk for heart failure at implant. There were three heart failure exacerbations and 17 treated ventricular arrhythmias. The study population-specific positive predictive value (PPV) of OptiVol was low (sensitivity $33 \%$ and $P P V \leq 4.4 \%$ ) for both CHF exacerbation and arrhythmias in all analyzed groups.

Conclusions: The sensitivity and positive predictive value of intrathoracic impedance monitoring was low in this population of adult congenital and pediatric patients. Recent improvements to the OptiVol algorithm may decrease these deficiencies. (PACE 2013; 36:994-999)

congestive heart failure, electrophysiology - clinical, defibrillation - ICD

Intrathoracic impedance (ITI) monitoring has recently been developed as a means to detect transpulmonary fluid accumulation in patients at risk for heart failure exacerbation. ${ }^{1}$ The goal of transthoracic impedance monitoring is to detect these physiologic changes before the patient develops clinical symptoms requiring acute management or hospitalization. An ITI monitoring program, OptiVol (Medtronic Inc., Minneapo-

Disclosures: LaPage has received honoraria from Medtronic for speaking about OptiVol. Serwer is a consultant to Medtronic. Bradley has received funding for fellowship training from Medtronic. Dick and von Alvensleben have no disclosures.

Address for reprints: Martin J. LaPage, M.D., Department of Pediatrics and Communicable Disease, Division of Pediatric Cardiology, University of Michigan, 1540 East Hospital Drive, Ann Arbor, MI 48109. Fax: 734-936-9470; e-mail: mlapage@med.umich.edu

Received October 24, 2012; revised January 3, 2013; accepted February 6, 2013.

doi: 10.1111/pace.12134 lis, MN, USA), has been incorporated into Medtronic implantable cardioverter-defibrillators (ICDs) since 2007. OptiVol makes daily ITI measurements, compares them to the individual patient's baseline impedance, and computes a continuous dynamic OptiVol fluid index value proposed to track changes in fluid content in lung and other thoracic tissues. ${ }^{2,3}$ Significant decreases in ITI values may indicate fluid accumulation and pending heart failure exacerbation. The trends of impedance measurements are available upon inoffice or remote device interrogation, allowing the care team to intervene before symptom onset.

ITI monitoring has been the subject of multiple trials of adult heart failure patients ${ }^{4-10}$ and additional publications in the past several years. ${ }^{11,12}$ These studies have generally shown fair sensitivity and fair to poor positive predictive values ranging from $5 \%$ to $60 \%$ and suggested a lower risk of heart failure hospitalization when using ITI monitoring in these early studies. Van Veldhuisen, however, showed a higher risk of hospitalization when utilizing ITI monitoring. ${ }^{13}$ Two additional studies suggested a possible 
association between ITI measurements and episodes of ventricular arrhythmia. ${ }^{14,15}$

This study examines the utility of ITI monitoring in a population of pediatric and congenital heart disease (CHD) patients. The primary goals of the analysis were to identify the sensitivity and predictive value of ITI monitoring in this unique population and to determine the association of ITI changes with arrhythmia in patients at significant risk for arrhythmia from cardiomyopathy or channelopathy.

\section{Methods}

A retrospective data analysis was performed on all patients with an OptiVol-capable implantable device. Entry into the study was the time of implant or establishment of care with the University of Michigan Congenital Heart Center. The endpoint was the latter of device explantation, exit from the University of Michigan Health system, or December 2010. Patients were excluded if they had an epicardial device or follow-up less frequently than once annually. OptiVol data from time of device implantation to December 31, 2010 were collected from CareLink (Medtronic Inc.) remote monitoring reports and in-office device interrogation. The OptiVol data were collected from the OptiVol Index report.

The OptiVol index (Fig. 1) is an easy to interpret continuous comparison of the daily ITI
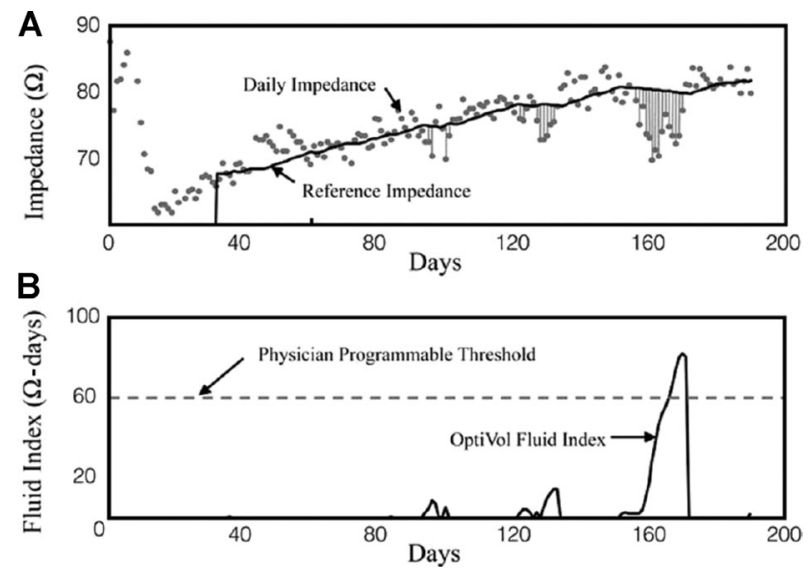

Figure 1. Diagram of impedance measures (top panel) and OptiVol index report (bottom panel) as provided in the device interrogation report. Daily impedance measures are compared to the reference impedance. Consecutive daily impedance values below the reference impedance will cause the fluid index to increase. The physician programmable threshold level is shown at the nominal 60 Ohm-days. OptiVol fluid index values crossing the threshold were considered positive for purposes of the study. Image used with permission from Medtronic Inc. measurement to the individual patient's dynamic baseline impedance. If the daily impedance is lower than the reference impedance, the OptiVol index value goes up. Continued increases in the OptiVol index value indicate continued decrease in the daily impedance measurement. Once the daily measure rises above the baseline, the index value returns to baseline. The OptiVol threshold, nominally set at 60 Ohm-days, is a physicianadjustable threshold value for the OptiVol Index indicating a possibly significant change in ITI. For each patient, a peak OptiVol index measurement (Ohm-days) was recorded for each half-month period of data and became a binned OptiVol data point. This peak measurement was recorded in increments of 20, with a range of $0-200$. The reasons for collecting OptiVol data in this way were threefold: (1) the OptiVol index report is easily accessible; (2) the OptiVol index report increments time into 1-month bins; these bins are easily divided in half for more frequent peak measurement recording; and (3) the OptiVol threshold is adjustable in increments of 20. All OptiVol measurement data were collected by a single individual blinded to other clinical data (Fig. 1).

Clinical data were collected from existing medical records and included basic demographics, cardiac diagnosis, indication for device implantation, New York Heart Association functional class, use of heart failure medications, and perceived baseline risk of heart failure based on structural anatomy. The primary indication for device implantation was considered to be arrhythmia in all patients with hypertrophic cardiomyoapathy, a genetic channelopathy, or documented ventricular arrhythmias before implant. For the purposes of the study, pediatric patients were defined as those patients entering the study at $\leq 16$ years of age. Patients were considered at risk for heart failure if they had documented chronic ventricular dysfunction by echocardiogram or a systemic right ventricle. The specific clinical outcome data collected were: occurrence of documented ventricular arrhythmias, delivered device therapies, and heart failure exacerbations which were qualified as documented change in medication or hospitalization for management of heart failure symptoms. All clinical data were collected by a separate individual blinded to the OptiVol data at the time of collection.

Binned OptiVol data points were associated with clinical events occurring (1) during the same or subsequent half-month bin and (2) during the two consecutive subsequent half-month bins. The rationale for this approach was to examine the validity of OptiVol changes to predict clinical events on a half-month or 1-month timeframe. Specificity and sensitivity of OptiVol were determined for 
both ventricular arrhythmia and heart failure exacerbation. Calculations for OptiVol to predict clinical outcome in the concurrent 1-month period or subsequent 1-month period were made based on the prevalence of the outcome in the study population. Separate analyses were performed to determine specificity, sensitivity, and positive predictive value in (1) the entire study cohort, (2) the subcohort of pediatric patients, and (3) the subcohort of patients at risk for heart failure. Separate analyses for sensitivity and populationspecific positive predictive values were calculated at incremental OptiVol threshold levels between 20 and $200 \mathrm{Ohm}$-days.

Statistical analysis included calculation of central tendency for continuous variables and proportions for categorical variables. Sensitivity, specificity, and positive predictive value were calculated using basic $2 \times 2$ tables of the study cohort. The Student's $t$-test was used to compare OptiVol Index measurements in patients with arrhythmia versus patients without arrhythmia. SAS 9.3 (Cary, NC, USA) was used for $t$-test calculations.

\section{Results}

Sixty-four OptiVol capable devices were implanted in 62 patients between January 2007 and September 2010. Seventeen devices were excluded from the analysis: four were epicardial implants and the remaining were not followed at the institution and had insufficient clinical follow-up records available for analysis. There were 47 patients (47 devices) included in the analysis: 18 in the CHF-risk subcohort and 23 in the pediatric subcohort. All included devices contained the original OptiVol program (not OptiVol 2.0 included in the Protecta XT device, Medtronic Inc.). Continuous OptiVol data from implant to study endpoint were available for 45 of 47 patients $(96 \%)$. Two patients had missing data points comprising $2 \%$ and $50 \%$ of their total binned data, respectively. Overall, there were 2,213 total binned OptiVol data points equivalent to 1,106 months of follow-up data; median follow-up time was 24 months per patient. The demographics and clinical characteristics of the total cohort and the subcohorts are shown in Table I. Overall $98 \%$ of patients had the device implanted primarily for arrhythmia. The pediatric subcohort included only one patient with a perceived risk of heart failure exacerbation. The prevalence of New York Heart Association class II heart failure or worse was only $17 \%$ in the total cohort and $39 \%$ in the CHF-risk subcohort. There were only two documented heart failure exacerbations during the study period, both occurring in patients in the CHF-risk group. There were three patients with documented arrhythmias in the CHF-risk subcohort and five in the pediatric subcohort.

Table I.

Demographic and Clinical Characteristics in Patients

\begin{tabular}{lccc}
\hline Variable & $\begin{array}{c}\text { All } \\
(\mathbf{n = 4 7 )}\end{array}$ & $\begin{array}{c}\text { CHF-Risk Subcohort } \\
(\mathbf{n}=\mathbf{1 8})\end{array}$ & $\begin{array}{c}\text { Pediatric Subcohort } \\
(\mathbf{n}=\mathbf{2 3})\end{array}$ \\
\hline Total OptiVol data: (months) & 1,106 & 445 & 526 \\
Age (years) at implant: mean (range) & $22(6-58)$ & $31(13-58)$ & $13(6-16)$ \\
Diagnosis: $\mathrm{n}(\%)$ & & & \\
$\quad$ TOF & $7(15)$ & $7(39)$ & 0 \\
TGA & $7(15)$ & $7(39)$ & 0 \\
Other CHD & $3(6)$ & $3(17)$ & $5(22)$ \\
LQTS & $10(21)$ & 0 & $7(30)$ \\
HCM & $7(15)$ & 0 & $5(17)$ \\
CPVT & $5(11)$ & 0 & $1(5)$ \\
DCM & $1(2)$ & $1(5)$ & $23(100)$ \\
Arrhythmia indication for implant: $\mathrm{n}(\%)$ & $46(98)$ & $17(94)$ & $1(5)$ \\
NYHA HF class $\geq 2: \mathrm{n}(\%)$ & $8(17)$ & $7(39)$ & $1(5)$ \\
\# on heart failure medications: $\mathrm{n}(\%)$ & $14(30)$ & $14(78)$ & $0(0)$ \\
\# with heart failure exacerbation: $\mathrm{n}(\%)$ & $1(2)$ & $1(6)$ & $5(22)$ \\
\# with arrhythmia events: $\mathrm{n}(\%)$ & $9(19)$ & $3(17)$ & 0
\end{tabular}

$\mathrm{CHD}=$ congenital heart disease; $\mathrm{CPVT}=$ catecholaminergic polymorphic ventricular tachycardia; $\mathrm{DCM}=$ dilated cardiomyopathy; $\mathrm{HCM}=$ hypertrophic cardiomyopathy; LQTS = Long QT syndrome; NYHA = New York Heart Association heart failure class; TGA = transposition of the great arteries; TOF = tetralolgy of Fallot. 
Table II.

OptiVol Predictability for Heart Failure Exacerbation

\begin{tabular}{lccccc}
\hline & No. of Events & $\begin{array}{c}\text { Threshold } \\
\text { Level }\end{array}$ & $\begin{array}{c}\text { No. of Threshold } \\
\text { Crossings }\end{array}$ & $\begin{array}{c}\text { Sensitivity \% } \\
\mathbf{( 9 5 \% ~ C l )}\end{array}$ & $\begin{array}{c}\text { Positive Predictive } \\
\text { Value \% (95\% Cl) }\end{array}$ \\
\hline Total cohort (A)* & 2 & 140 & 62 & $33(6-76)$ & $3.3(0.6-12)$ \\
Total cohort (B) & 2 & 80 & 131 & $33(6-76)$ & $1.5(0.3-6)$ \\
CHF-risk subcohort (A)* & 2 & 80 & 93 & $33(6-76)$ & $2.2(0.4-8)$ \\
\hline
\end{tabular}

$(\mathrm{A})^{\star}=$ OptiVol measurement correlated to event during concurrent and subsequent half-month period; $(\mathrm{B})^{\star}=\mathrm{OptiVol}$ measurement correlated to event during consecutive half periods after OptiVol data point. The pediatric subcohort had no events. CHF $=$ congestive heart failure; $\mathrm{Cl}=$ confidence interval.

Table III.

OptiVol Predictability for Ventricular Arrhythmia

\begin{tabular}{lccccc}
\hline & No. of Events & $\begin{array}{c}\text { Threshold } \\
\text { Level }\end{array}$ & $\begin{array}{c}\text { No. of Threshold } \\
\text { Crossings }\end{array}$ & $\begin{array}{c}\text { Sensitivity \% } \\
\mathbf{( 9 5 \% ~ C l )}\end{array}$ & $\begin{array}{c}\text { Positive Predictive } \\
\text { Value \% (95\% Cl) }\end{array}$ \\
\hline Total cohort (A)* & 17 & 20 & 784 & $31(17-48)$ & $1.4(0.7-2.5)$ \\
Total cohort (B) & 17 & 20 & 784 & $29(15-47)$ & $1.3(0.6-2.4)$ \\
CHF-risk subcohort (A)* & 9 & 20 & 370 & $30(14-53)$ & $1.9(0.8-4)$ \\
Pediatric subcohort (A)* & 7 & 20 & 290 & $29(1-58)$ & $1.4(0.4-4)$
\end{tabular}

$(\mathrm{A})^{\star}=$ OptiVol measurement correlated to event during concurrent and subsequent half-month period; $(\mathrm{B})^{\star}=\mathrm{OptiVol}$ measurement correlated to event during consecutive half. $\mathrm{CHF}=$ congestive heart failure; $\mathrm{Cl}=$ confidence interval.

The sensitivities and population-specific positive predictive values were calculated at serial OptiVol thresholds $(20,40,60, \ldots, 200)$. Because 70 different analyses were performed, only the OptiVol thresholds that produced the highest positive predictive value for each subcohort analysis are reported in Tables II and III. Also included in Tables II and III are the total number of threshold crossings for each analysis. OptiVol was poorly predictive of both heart failure exacerbations and arrhythmias in this population of patients; this was largely a consequence of the low incidence of the clinical outcome.

\section{Discussion}

This study revealed several important findings regarding the utility of ITI monitoring. First, the positive predictive value in patients considered to be at risk for heart failure exacerbation was quite low. Second, the incidence of heart failure exacerbations in this population of all patients with an OptiVol-capable ICD from a single center was also low. Finally, no relationship could be found between ITI monitoring and arrhythmia occurrence.

The positive predictive value of a test is in part a function of the prevalence of disease. Consider- ing the population as a whole, the incidence of pediatric heart failure as indicated by a diagnosis of dilated cardiomyopathy $(0.34$ per 100,000$)$ is a tiny fraction of the incidence of congestive heart failure in the adult population between ages 60 and 80 years $(7,200$ per 100,000$) \cdot{ }^{16}$ In addition, the majority of the adult population with ICDs has them implanted for congestive heart failure. ${ }^{17}$ The pediatric and CHD population, on the other hand, more commonly have ICDs implanted for genetic channelopathies, hypertrophic cardiomyopathy, and ventricular arrhythmias subsequent to repaired CHD. ${ }^{18-20}$ There are no specific recommendations or conclusive evidence that ICDs should be implanted for primary prevention in pediatric congestive heart failure.

Aside from these factors, the incidence of heart failure exacerbation in pediatric patients overall should be considered when determining the true utility of an ITI monitoring device. Webster et al. ${ }^{21}$ performed a national database study that accounted for $50 \%$ of the pediatric hospitalizations in the United States in 1997. Of these, 5,600 patients had a diagnosis of heart failure suggesting a possibility of 11,000 children hospitalized for heart failure each year. However, $56 \%$ of these patients were infants, and heart failure was only the primary diagnosis in $25 \%$ 
of the patients. This indicates that the more realistic number of potentially preventable heart failure hospitalizations is only 1,200 per year if all patients had an ITI monitoring-capable device with $100 \%$ sensitivity to predetect heart failure exacerbation.

CHD patients specifically have anatomical constraints to ITI monitoring reliability as the system is designed to measure thoracic fluid accumulation occurring with systemic ventricular failure. Patients with a Fontan circulation will almost always have an epicardial device. The utility of impedance monitoring between an epicardial coil and an abdominal can is not known. Patients with repaired tetralogy of Fallot are more subject to right heart failure exacerbation, and transpulmonary impedance may not change appreciably. The primary adult CHD patient may benefit from ITI monitoring in the patient with a systemic right ventricle after atrial switch operation for transposition of the great arteries, as heart failure in these patients is an almost expected course. The number of patients with this specific diagnosis was small, however, and larger studies may show improved value.

Prior studies have suggested a possible association between ITI and occurrence of arrhythmia. ${ }^{14,15}$ This study did not find such an association, though the precision with which changes in Ohm-day values were determined was much lower than in these prior studies. Moore et al. ${ }^{14}$ showed that $64 \%$ of ventricular tachycardia/ventricular fibrillation episodes were preceded by a mean impedance decrease of 4 Ohms. This study would not have detected these effects because changes in ITI were determined from the indexed data and not from the exact impedance measures. The precision specificity of such small changes would also be expected to compromise their predictive value.

Prior adult studies have shown a significant risk of false positive events. Medtronic literature on $\mathrm{OptiVol}^{3}$ and a prior publication ${ }^{11}$ have provided much information on possible causes of false positive events. Despite these, the majority of false positive events have been of unknown etiology - potentially subclinical episodes of heart failure exacerbation that resolve spontaneously or normal physiologic variation. This study population provides a unique opportunity to see the risk of false positive events in a population of patients with no risk for CHF exacerbation. For example, in the pediatric group, a nominal

\section{References}

1. Wang L, Lahtinen S, Lentz L, Rakow N, Kaszas C, Ruetz L, Stylos L, et al. Feasibility of using an implantable system to measure thoracic
OptiVol threshold level of 60 resulted in 205 $(10 \%)$ of the 2,213 total binned OptiVol index values being above the threshold and therefore a false positive event.

OptiVol technology evolved during this study. All devices included in this study utilized the original OptiVol programming algorithms. The new OptiVol 2.0, included in the Medtronic Protecta devices, uses modified algorithms to determine the reference and index values to improve sensitivity and decrease false positives. ${ }^{22}$ A detailed description of these changes is included in Von Bergen et al. ${ }^{20}$ How these changes will affect the utility in pediatrics and CHD is unclear. Despite the change in technology, this study does help outline some of the problems with ITI monitoring in this population. OptiVol 2.0 is currently only available in the Protecta XT device and may come at higher cost; pediatric electrophysiologists will need further evidence to decide if the potential benefit of OptiVol monitoring is worth the higher cost.

\section{Limitations}

This was a retrospective data set. Mild heart failure exacerbations or medication changes may have been missed, although the importance of missing episodes that did not result in hospitalization is not convincing. The ITI data were collected using the OptiVol index report, because this report is easily interpretable and obtainable by the physician. It is possible that more significant findings would have been discovered if raw impedance measurement data were obtained. The occurrence of the clinical endpoint was very small.

\section{Conclusions}

In this population of pediatric and CHD patients, ITI analysis was poorly predictive of clinical heart failure or arrhythmia events, due to low sensitivity and very low prevalence of these clinical events in the study population. The best positive predictive value determined was $4.4 \%$ for patients with a systemic right ventricle (OptiVol Index threshold set at $80 \mathrm{Ohm}$-days), though the number of these patients was small. The current version of OptiVol was not available at the time of this study, and improvements in the current algorithms may significantly improve the functionality in all patients. Ideally, prospective studies will be needed to determine efficacy and validity of the system in pediatric and CHD patients. 


\section{PEDIATRIC/CONGENITAL INTRATHORACIC IMPEDANCE MONITORING}

2. Wang L. Fundamentals of intrathoracic impedance monitoring in heart failure. Am J Cardiol 2007; 99:3G-10G.

3. Medtronic Connect Website. https://wwwp.medtronic.com/mdtCon nectPortal/dynamicarea/optivolpredictsworseningheartfailure/1581 802169585. Accessed September 16, 2012.

4. Yu C-M, Wang L, Chau E, Chan RH-W, Kong S-L, Tang $\mathrm{M}-\mathrm{O}$, Christensen J, et al. Intrathoracic impedence monitoring in patients with heart failure: Correlation with fluid status and feasibility of early warning preceding hospitalization. Circulation 2005; 112:841-848.

5. Ypenburg C, Bax JJ, van der Wall EE, Schalij MJ, van Erven L. Intrathoracic impedance monitoring to predict decompensated heart failure. Am J Cardiol 2007; 99:554-557.

6. Vollmann D, Nagele H, Schauerte P, Weigand U, Butter C, Zanotto G, Quesada A, et al. Clinical utility of intrathoracic impedance monitoring to alert patients with an implanted device of deteriorating chronic heart failure. Eur Heart J 2007; 28:18351840 .

7. Maines M, Catanzariti D, Cemin C, Vaccarini C, Vergara G. Usefulness of intrathoracic fluids accumulation monitoring with an implantable biventricular defibrillator in reducing hospitalizations in patients with heart failure: A case-control study. J Interv Card Electrophysiol 2007; 19:201-207.

8. Catanzariti D, Lunati M, Landolina M, Zanotto G, Lonardi G, Iacopino S, Oliva F, et al. Monitoring intrathoracici impedance with an implantable defibrillator reduces hospitalizations in patients with heart failure. Pacing Clin Electrophysiol 2009; 32 : 363-370.

9. Whellan DJ, Ousdigian KT, Al-Khatib SM, Pu W, Sarkar S, Porter CB, Pavri BB, et al. Combined heart failure device diagnostics identify patients at higher risk of subsequent heart failure hospitalizations: Results from PARTNERS HF (Program to Access and Review Trending information and Evaluate correlation to Symptoms in patients with Heart Failure) study. J Am Coll Cardiol 2010; 55:18031810.

10. Conraads VM, Tavazzi L, Santini M, Oliva F, Gerritse B, Yu CM, Cowie MR. Sensitivity and positive predictive value of implantable intrathoracic impedance monitoring as a predictor of heart failure hospitalizations. Eur Heart J 2011; 32:2266-2273.

11. Wang L. Key lessons from cases worldwide. Am J Cardiol 2007; 99:34G-40G.
12. Small RS. Integrating device-based monitoring into clinical practice: Insights from a large heart failure clinic. Am J Cardio 2007; 99:17G-22G.

13. Van Veldhuisen DJ, Braunschweig F, Conraads V, Ford I, Cowie MR, Jondeau G, Kautzner J, et al. Intrathoracic impedance monitoring, audible patient alerts, and outcome in patients with heart failure. Circulation 2011; 124:1719-1726.

14. Moore HJ, Peters MN, Franz MR, Karasik PE, Singh SN, Fletcher RD. Intrathoracic impedance preceding ventricular tachyarrhythmia episodes. Pacing Clin Electrophysiol 2010; 33:960-966.

15. Ip JE, Cheun JW, Park D, Hellawell, JL, Stein KM, Iwai S, Liu $\mathrm{CF}$, et al. Temporal associations between thoracic volume overload and malignant ventricular arrhythmias: A study of intrathoracic impedance. J Cardiovasc Electrophysiol 2011; 22:293-299.

16. Lipshultz SE, Sleeper LA, Towbin JA, Lowe AM, Orav EJ, Cox GF, Lurie PR, et al. The incidence of pediatric cardiomyopathy in two regions of the United States. N Engl J Med 2003; 348:1647-1655.

17. Hammill SC, Kremers MS, Stevenson LW, Heidenreich PA, Lang CM, Curtis JP, Wang Y, et al. Review of the Registry's Fourth Year, incorporating lead data and pediatric ICD procedures, and use as a national performance measure. Heart Rhythm 2010; 7:1349-1345.

18. Burns KM, Evans F, Kaltman JR. Pediatric ICD utilization in the United States from 1997 to 2006. Heart Rhythm 2011; 8:23-28.

19. Berul CI, Van Hare GF, Kertesz NJ, Dubin AM, Cecchin F, Collins KK, Cannon BC, et al. Results of a multicenter retrospective implantable cardioverter-defibrillator registry of pediatric and congenital heart disease patients. J Am Coll Cardiol 2008; 51:16851691.

20. Von Bergen NH, Atkins DL, Dick M 2nd, Bradley DJ, Etheridge SP, Saaral EV, Fischbach PS, et al. Multicenter study of the effectiveness of implantable cardioverter defibrillators in children and young adults with heart disease. Pediatr Cardiol 2011; 32:399-405.

21. Webster G, Zhang J, Rosenthal D. Comparison of the epidemiology and co-morbidities of heart failure in the pediatric and adult populations: A retrospective, cross-sectional study. BMC Cardiovasc Disord 2006; 6:23-30.

22. Sarkar S, Hettrick DA, Koehler J, Rogers T, Gringerg Y, Yu C-M, Abraham WT, et al. Improved algorithm to detect fluid accumulation via intrathoracic impedance monitoring in heart failure patients with implantable devices. J Cardiac Failure 2011; $17: 569-576$ 